\title{
Article \\ Agrochemicals used in freshwater aquaculture in Jhenaidah district, Bangladesh
}

\author{
Md. Shahinur Rahman ${ }^{1}$, Subrata Mondal ${ }^{1 *}$ and Amir Hossain ${ }^{2}$ \\ ${ }^{1}$ Department of Fisheries and Marine Bioscience, Faculty of Biological Science and Technology, Jashore \\ University of Science and Technology, Jashore-7408, Bangladesh \\ ${ }^{2}$ International Studies of Aquatic Tropical Ecology, Department of Biology, University of Bremen, \\ Bibliothekstraße 1, 28359 Bremen, Germany
}

*Corresponding author: Subrata Mondal, Department of Fisheries and Marine Bioscience, Faculty of Biological Science and Technology, Jashore University of Science and Technology, Jashore-7408, Bangladesh. E-mail: subrata.fmb.just@gmail.com

Received: 14 October 2019/Accepted: 20 November 2019/ Published: 30 November 2019

\begin{abstract}
The present experiment was conducted in order to investigate the use of agrochemicals in freshwater aquaculture with emphasis on fish health management. The selected area was Mahespur upazila under Jhenaidah district and the study duration was six months. The main group was fifty fish farmers and the data were collected through interview with fish farmers and key informants (chemical seller). Ten categories of chemicals were identified that are used by fish farmers for various purposes such as pond preparation, water quality management, controlling diseases, supplying oxygen, killing fish predators, Ten pharmaceutical companies were found to provide the agrochemicals to fulfill the farmers need. These companies are Novartis Anmal Health Ltd, Fishtech (BD) Ltd, SK+F BD Ltd, Renata Ltd, ACI Animal Health, Opsonin Pharma Ltd, Eon Animal Health Products Ltd, Square Pharmaceuticals Ltd, Sciencetech Agro Industries Ltd, Rals. In the study area some kinds of diseases were founded such as Epizotic Ulcerative Syndrome (EUS) in Rohu (Labeo rohita), Catla (Catla catla), Mozambique tilapia (Oreochromis niloticus), Black spot in Stinging cat fish, skin diseases, gill damage, tail and fin rot in Yellow tail catfish (Pangasius pangasius). For highest stocking density tilapia and pangus were mostly affected by diseases in winter. Farmers use various chemicals such as for water quality management agriculture lime, Geotox, JV Zeolite, Mega Zeo Bio, Aquakleen and Biomin, as antibiotic they use Novamix 101, Erocot, Captor, Oxysentin 20\%, Renamycin, Aquamycin and Oxysentin 20\% are antibiotics with different trade names were seen in the market as well as used by the fish farmers in the study area. The fish farmers use a wide variety of disinfectants in freshwater aquaculture. Timsen, Polgard plus, Formalin, Bleaching powder, EDTA are found available in all the shops of Mahespur. Formalin is used to control protozoan diseases. Virex is used to destroy virus and bacteria. Fish farmers use oxygen suppliers to increase the oxygen level in the water body. Oxidizing agent, hydrogen peroxide are major active ingredients of such chemical. The recovery of fish diseases was found $80-90 \%$ in the study area. During field observation many problems were identified in case of using agrochemicals which included lack of skill and knowledge of farmers about the application, withdrawal period of agrochemicals and drugs and some adverse effects on fish and human health.
\end{abstract}

Keywords: aqua medicine; agrochemicals; inland aquaculture; Jhenaidah district; Bangladesh

\section{Introduction}

In Bangladesh the production of aquaculture is increasing day by day through diversification (Mahmud et al., 2012). In the past, people depended mainly on wild fishes and other natural resources but catches have declined due to increased fishing effort and range of other anthropogenic pressures (Apu, 2014). As a result, aquaculture has grown rapidly to fill the gap. Maheshpur is an Upazila under Jhenaidah District. Fish culture mainly dependent on the input of formulated feeds and the application of agro chemicals. But different types of diseases 
could be found in farmed aquatic animals in Bangladesh (Karim and Stellwagen, 1998; BFRI, 1999; Faruk et al., 2004a). A number of diseases like Epizootic Ulcerative Syndrome (EUS), skin erosion, gill damage, tall and fin rot are common in farmed fishes of Bangladesh (Faruk et al., 2004b). To cure disease and maintain proper health condition of fish agrochemicals play a vital role. Chemicals are indeed an essential ingredient to successful aquaculture, which has been used in various forms for centuries (Subasinghe et al., 1996). Chemicals and antibiotics are important components in health management of aquatic animals, pond construction, soil and water management, improving aquatic productivity, transportation of live fish, feed formulation, manipulation of reproduction, growth, processing and value addition of the final product (Subasinghe et al., 1996). In aquaculture, chemicals can be classified by the purpose of uses, the type of organisms under culture, the life cycle stage for which they are used, the culture system, intensity of culture and by the type of people who use them (Primavera et al., 1993). The world agrochemicals is a general term used to refer to chemicals that are employed to control, destroy, mitigate, prevent or repel pests on agricultural products (Meisinger and Delago, 2002). Agrochemicals include pesticides, lime, antibiotic, hormones etc. Commonly used chemicals in Bangladesh aquaculture are lime, rotenone, various forms of inorganic and organic fertilizers, phostoxin, salt, dipterex, antimicrobials, potassium permanganate, copper sulphate, formalin, sumithion, melathion etc. (Brown and Brooks, 2002; Faruk et al., 2005). Although aquaculture industry is growing in Mahespur upazila, Jhenaidah, Bangladesh, and a large number of aqua chemicals are being used by the farmers, but no previous systematic investigation were conducted to evaluate the present status of agrochemicals used in aquaculture in this present study area. Considering above mentioned prospects and knowledge gap, this study was conducted to identify different types of agrochemicals used in aquaculture in the study area and to know the purposes, dose and mode of application of the used agrochemicals.

\section{Materials and Methods}

\subsection{Study area and period of study}

The study was carried out from November 2018 to May 2019 on the fishing community of Mahespur upazila under Jhenaidah district. The area is located between $23^{\circ} 21^{\prime}$ to $23.35^{\circ}$ north latitude and $88^{\circ} 54.8^{\prime}$ to $88.9133^{\circ}$ east longitude.

\subsection{Selection of target group and sample size}

The target group was fishermen who were involved in using agro chemicals using for fish health management permanently (as the primary income source) and partially (as the secondary income source) from aquaculture. The data were collected randomly from 50 fishermen and agrochemicals seller.

\subsection{Data collection}

Primary data were collected by field survey and selection of fishermen, questionnaire interview, and Focus Group Discussion (FGD). Secondary data were collected from various books, reports, journals, bulletins, thesis paper and organizations.

\subsection{Data processing and analysis}

After data collection, these were verified to eliminate errors and inconsistencies. Any kind of inconsistencies in collected data were searched and discarded from the data. Data were processed and finally analyzed using tabular method. The data of local units were converted into international unit before analysis. The data were calculated by Microsoft Excel 2010.

\section{Results}

\subsection{Basic profile of the fishermen}

\subsubsection{Family size and type}

The result showed about 70\% families were small (2-4 person), 24\% families were medium (5-7 person) and 6\% families were large ( 8 person and above) (Figure 1). 


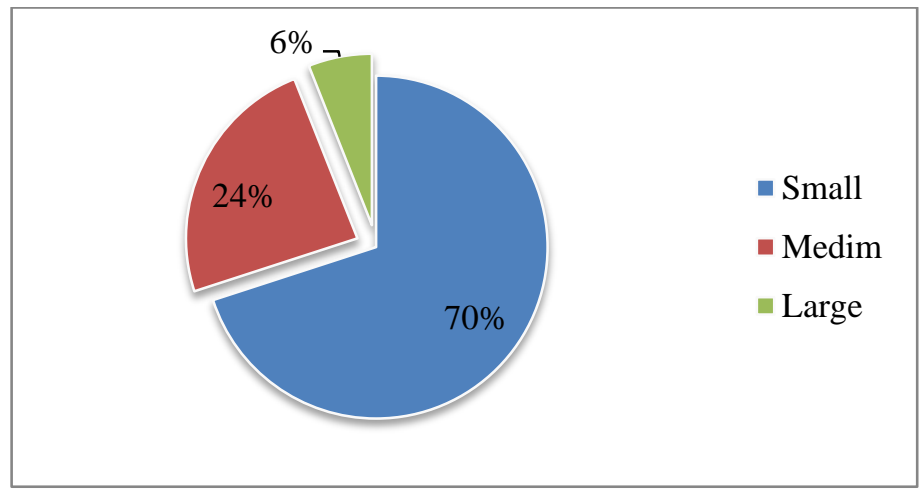

Figure 1. Family size of the respondents.

\subsubsection{Age of the fish farmers}

About $18 \%$ of the fish farmers were less than 26 years and 2\% were 15-18 years old. They are divided into three categories. Young (18-25) years, middle (26-45) years and old above 45 years (Figure 2).

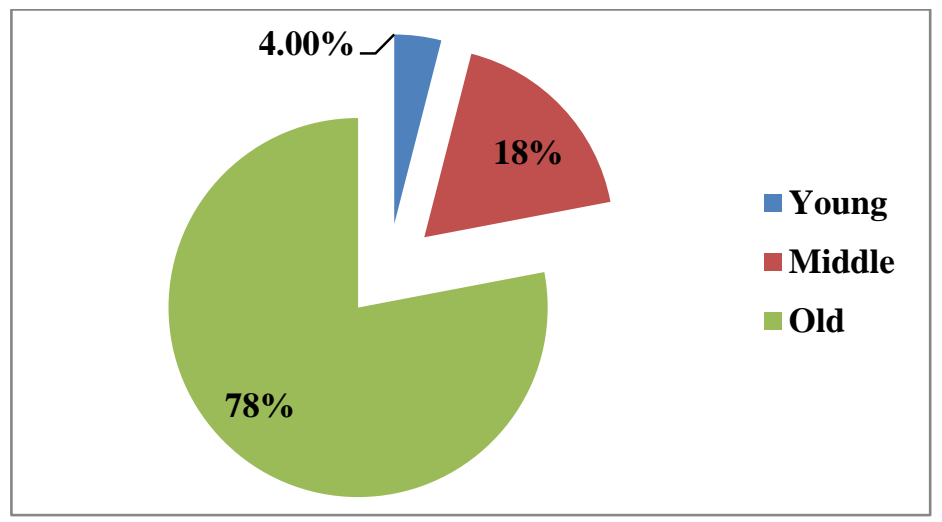

Figure 2. Age categories of the respondents.

\subsubsection{Educational status}

About $6 \%$ fish farmer had no education. About 35\% people can only sign only and $80 \%$ were gained their primary education only. $5 \%$ fish farmer had education up to S.S.C level only. Between them $4 \%$ fish farmer had gained higher education and acquired bachelor degree (Figure 3).

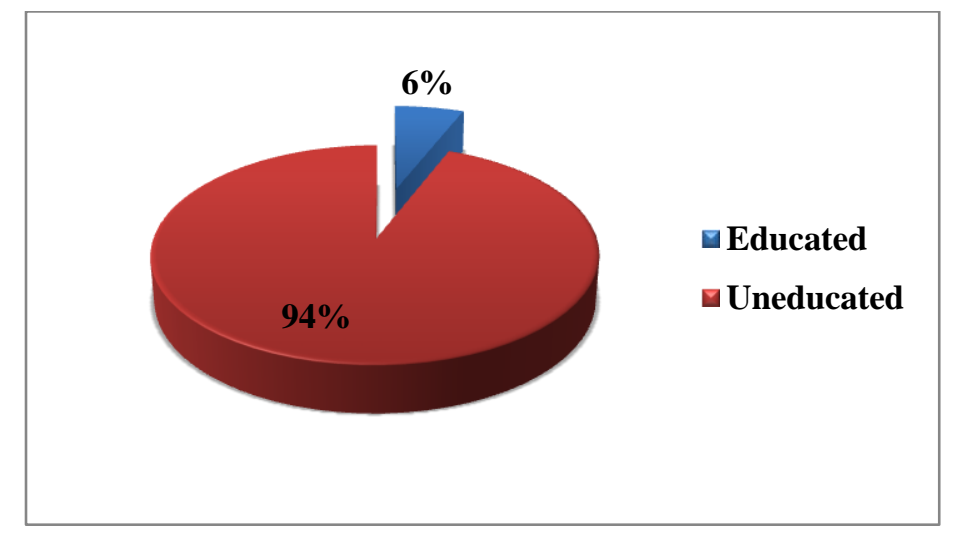

Figure 3. Educational status of the respondents.

\subsubsection{Pond ownership and land holding status}

Among 50 fishermen of my study areas about $75 \%$ farmers having single ownership and $25 \%$ farmers having multiple ownership. About $82 \%$ fishermen had own land and the other farmer about $18 \%$ had culture others land (Table 1). 
Table 1. The land holding statuses of farmer.

\begin{tabular}{|l|l|l|l|}
\hline Categories & Min (ha) & Max (ha) & Mean \pm SD \\
\hline Small (.6-2.67) ha & 0.6 & 2.67 & $1.613 \pm 0.536$ \\
\hline Medium(2.81-6.69) ha & 2.81 & 6.69 & $4.478 \pm 1.104$ \\
\hline Large(6.82-12.04) ha & 6.82 & 12.04 & $9.508 \pm 2.130$ \\
\hline
\end{tabular}

\subsubsection{Occupation}

The fishermen of my study area had more than two occupations. They all were not involved with fish culture completely. Fish culture was the main occupation of the maximum fishermen the percentage was about $94 \%$. Besides fishing some people involved with other profession as their secondary occupation. The percentage of these kinds fishermen was about $6 \%$. Some fishermen found in the study areas that they become involved with the secondary occupation when primary occupation means fishing cannot provide full time employment. Most of them were involved with agriculture besides of fishing.

\subsubsection{Sources of credit}

In my study areas it was found that about $76 \%$ fishermen used their own money for their culture practice. About $8 \%$ of them received credit facilities from the bank and others were involved with different NGOs for their credit facilities purposes. The amount of their loan was minimum 10,000BDT to 60,000 BDT respectively. The mean of loan was $32083.33 \pm 15733.33$. They had to pay the extra interest was about 650BDT to 9,000BDT.

\subsubsection{Training status}

About $75 \%$ fishermen of my study areas had no formal training. They were gained experience by self-practice. About 25\% fishermen were getting formal training from the Upazila Fishery office with the help of Department of Fisheries (DoF).

\subsubsection{Crop farming status}

About 6\% fishermen were involved with crop farming besides of fish culture. Maximum of them were small fishermen. They were gross paddy only Boro season. The cultivated land were recorded that minimum 0.6 ha to 1.6 ha. The mean of cultivated areas was $0.98 \pm 0.54$ and production $4278.33 \pm 1492.37$. The average cultivation cost was $431666.67 \pm 33579.51$. The mean gross income per ha was 59083. \pm 45989.35 . The data are given in the (Table 2).

Table 2. Crop farming status of farmer.

\begin{tabular}{|l|l|l|l|}
\hline Issue & Min & Max & Mean \pm SD \\
\hline Cultivated area (ha) & 0.6 & 1.6 & $0.98 \pm 0.54$ \\
\hline Cultivation cost(BDT/ha) & 4500 & 65000 & $431666.67 \pm 33579.51$ \\
\hline Production (kg/ha) & 2560 & 5250 & $4278.33 \pm 1492.37$ \\
\hline Gross income(BDT/ha) & 7250 & 95000 & $59083 \pm 45989.35$ \\
\hline
\end{tabular}

\subsubsection{Household income and expenditures}

In my study areas it was found that the HH income of the fishermen was minimum 15,000BDT and maximum $1,67,000$ BDT. The mean of the amount was 52520 442408.24 BDT (Table 3).

Table 3. Household income and expenditures of the respondents.

\begin{tabular}{|l|l|l|l|l|}
\hline Expenditure issue & \multirow{2}{*}{ HH (\%) } & \multicolumn{3}{|c|}{ Cost (BDT/year) } \\
\cline { 2 - 5 } & & Min & Max & Mean \pm SD \\
\hline Food & 100 & 8500 & 75000 & $38060 \pm 20714.19$ \\
\hline Education & 60 & 1000 & 30000 & $14640 \pm 11135.08$ \\
\hline Health & 100 & 1000 & 5000 & $2458 \pm 1857.92$ \\
\hline Cloths & 100 & 2000 & 25000 & $8800 \pm 7886.96$ \\
\hline Furniture & 100 & 1000 & 70000 & $26340 \pm 24701.98$ \\
\hline Others & 100 & 7000 & $3600 \pm 2626.39$ \\
\hline
\end{tabular}


3.2. Agrochemicals used by fish farmers in Maheshpur

3.2.1. Chemicals for pond preparation and water quality management

For water quality management agriculture lime, Geotox, JV Zeolite, Mega Zeo Bio, Aquakleen and Biomin pond life have been used in the study area (Table 4).

Table 4. List of water management chemicals used in pond.

\begin{tabular}{|c|c|c|c|c|}
\hline Trade Name & Active ingredients & Dose & Source & Price (Taka) \\
\hline $\begin{array}{l}\text { Agricultural } \\
\text { lime }\end{array}$ & $\mathrm{CaCO}_{3}$ & spread with water6-10 ppm & Chemical seller & $8-15 / \mathrm{kg}$ \\
\hline Mega Zeo Bio & $\begin{array}{l}\mathrm{SiO}_{2}, \mathrm{Al}_{2} \mathrm{O}_{3}, \mathrm{CaO}, \mathrm{MgO}, \\
\mathrm{Fe}_{2} \mathrm{O}_{3}, \mathrm{Na}_{2} \mathrm{O}\end{array}$ & $200 \mathrm{gm} / \mathrm{dec}$ & ACI Animal Health & $340 / 10 \mathrm{~kg}$ \\
\hline Geotox & $\begin{array}{l}\mathrm{SiO}_{2}, \mathrm{Al}_{2} \mathrm{O}_{3}, \mathrm{CaO}, \mathrm{MgO}, \\
\mathrm{Fe}_{2} \mathrm{O}_{3}, \mathrm{Na}_{2} \mathrm{O}\end{array}$ & $\begin{array}{l}\text { For 3-6 feet deep water } 20-25 \\
\mathrm{~kg} / \mathrm{dec} \text { about } 30-40 \text { days }\end{array}$ & $\begin{array}{l}\text { Noverties Animal } \\
\text { Health Products Ltd. }\end{array}$ & $55 / \mathrm{kg}$ \\
\hline JV zeolite & $\begin{array}{l}\mathrm{SiO}_{2}, \mathrm{Al}_{2} \mathrm{O}_{3}, \mathrm{CaO}, \mathrm{MgO} \\
\mathrm{Fe}_{2} \mathrm{O}_{3}, \mathrm{Na}_{2} \mathrm{O}, \mathrm{K}_{2} \mathrm{O}, \mathrm{Mn}, \mathrm{P}\end{array}$ & $6-7 \mathrm{~kg} / 33 \mathrm{dec}$ up to mud & $\begin{array}{l}\text { Eon Animal Health } \\
\text { Products Ltd. }\end{array}$ & $350 / 10 \mathrm{~kg}$ \\
\hline $\begin{array}{l}\text { Biomin pond } \\
\text { life }\end{array}$ & Probiotics & 200/Bigha & Renata Ltd. & $2100 / \mathrm{kg}$ \\
\hline
\end{tabular}

\subsubsection{Antibiotics}

All of these antibiotics are effective against bacterial disease. These broad spectrum antibiotics were found to use by mixing with fish feed. The list of antibiotics is given in Table 5 .

Table 5. The list of antibiotics used in pond of study area.

\begin{tabular}{|c|c|c|c|c|c|}
\hline Trade name & Active ingredients & Purposes & Dose & Source & Price (Taka) \\
\hline Aquamycin & Oxytetracyclin HCL $25 \%$ & $\begin{array}{l}\text { To reduce } \\
\text { mortality, To help } \\
\text { retaining color of } \\
\text { fishes }\end{array}$ & $1-2 \mathrm{~g} / \mathrm{Kg}$ feed & $\begin{array}{l}\text { ACI Animal } \\
\text { Health }\end{array}$ & $70 / 100 \mathrm{~g}$ \\
\hline $\begin{array}{l}\text { Renamycin } \\
\text { soluble powder }\end{array}$ & Oxytetracyclin $200 \mathrm{mg}$ & $\begin{array}{l}\text { To improve } \\
\text { immune system } \\
\text { of fishes }\end{array}$ & $\begin{array}{l}50 \mathrm{mg} / \mathrm{Kg} \\
\text { fish body } 10 \\
\text { days }\end{array}$ & Renata Ltd. & $820 / \mathrm{kg}$ \\
\hline Novamix 101 & Doxycyclin & $\begin{array}{l}\text { Effective for } \\
\text { streptococcus } \\
\text { infection }\end{array}$ & $2-3 \mathrm{~g} / \mathrm{kg}$ feed & $\begin{array}{l}\text { Fishtech (BD) } \\
\text { Ltd. }\end{array}$ & $37 / 100 \mathrm{~g}$ \\
\hline Neoxel & Neomycinesulphate & $\begin{array}{l}\text { Effective for all } \\
\text { gram posiive } \\
\text { gram negative } \\
\text { bacteria }\end{array}$ & $1 \mathrm{~g} / 20 \mathrm{~kg}$ feed & $\begin{array}{l}\text { SK+F BD } \\
\text { Ltd. }\end{array}$ & $290 / 100 \mathrm{~g}$ \\
\hline Novamix 103 & Erythromycin & $\begin{array}{l}\text { To kill the aquatic } \\
\text { harmful germ }\end{array}$ & $2-3 \mathrm{~g} / \mathrm{feed}$ & $\begin{array}{l}\text { Fishtech (BD) } \\
\text { Ltd. }\end{array}$ & $330 / 100 \mathrm{~g}$ \\
\hline Captor & Chlortetracycline HCL $45 \%$ & $\begin{array}{l}\text { To increase fish } \\
\text { production, To } \\
\text { improve immune } \\
\text { system of fishes }\end{array}$ & $\begin{array}{l}50-70 \mathrm{~g} / 100 \\
\mathrm{Kg} \text { feed for } 5- \\
7 \text { days }\end{array}$ & $\begin{array}{l}\text { Novartis } \\
\text { Anmal Health } \\
\text { Ltd. }\end{array}$ & $405 / 100 \mathrm{~g}$ \\
\hline Oxysent $20 \%$ in & Oxytetracyclin HCL $200 \mathrm{mg}$ & $\begin{array}{l}\text { Most effective in } \\
\text { the treatment of } \\
\text { EUS }\end{array}$ & $\begin{array}{l}100-200 \\
\mathrm{~g} / 100 \mathrm{~kg} \text { feed } \\
\text { for 5-7 days }\end{array}$ & $\begin{array}{l}\text { Novartis } \\
\text { Anmal Health } \\
\text { Ltd. }\end{array}$ & $840 / 1 \mathrm{~kg}$ \\
\hline Erocot & Erythromycin+Sulphodiazine & $\begin{array}{l}\text { To improve } \\
\text { immune system } \\
\text { of fishes }\end{array}$ & $\begin{array}{l}1 \mathrm{~g} / 10 \mathrm{~kg} \text { feed } \\
\text { for } 2-5 \text { days }\end{array}$ & $\begin{array}{l}\text { Opsonin } \\
\text { Pharma Ltd. }\end{array}$ & 420 \\
\hline
\end{tabular}

\subsubsection{Disinfectants}

Timsen, Polgard plus, Formalin, Bleaching powder, EDTA are found available in all the shops of Mahespur. The list of disinfectant is given in Table 6. 
Table 6. List of disinfectant used in pond of study area.

\begin{tabular}{|l|l|l|l|l|l|}
\hline Trade name & Active ingredients & Purposes & Dose & Source & Price (Taka) \\
\hline Timsen & $\begin{array}{l}\text { n-alkyl dimethyl benzyl } \\
\text { ammonium chloride- } \\
40 \%, \text { Stabilized Urea- } \\
60 \%\end{array}$ & $\begin{array}{l}\text { Quickly eliminate } \\
\text { micro organisms in } \\
\text { fish ponds }\end{array}$ & $\begin{array}{l}80 \mathrm{~g} / 33 \text { decimal } \\
\left(1^{\text {st }} \text { dose }\right) \\
50 \mathrm{~g} / 33 \text { decimal } \\
\left(2^{\text {nd }} \mathrm{dose}\right)\end{array}$ & $\begin{array}{l}\text { Eon Animal } \\
\text { Health } \\
\text { Products } \\
\text { Ltd. }\end{array}$ & 260 \\
\hline Formalin & $40 \%$ Formaldehyde & $\begin{array}{l}\text { To remove } \\
\text { infectious disease }\end{array}$ & $1-3 \mathrm{ppm}$ & $\begin{array}{l}\text { Chemical } \\
\text { seller }\end{array}$ & $70 / \mathrm{kg}$ \\
\hline $\begin{array}{l}\text { Bleaching } \\
\text { Power }\end{array}$ & Chlorine & $\begin{array}{l}\text { To kill virus } \\
\text { microbes }\end{array}$ & $60 \mathrm{ppm}$ & $\begin{array}{l}\text { Chemical } \\
\text { seller }\end{array}$ & $50 / \mathrm{kg}$ \\
\hline Virex & $\begin{array}{l}\text { Potassium peroxymono } \\
\text { sulphate 50\% }\end{array}$ & $\begin{array}{l}\text { To protect from } \\
\text { bacteria and virus, } \\
\text { To protect from } \\
\text { black gill, tail,rot } \\
\text { and lesion }\end{array}$ & $\begin{array}{l}150-200 \mathrm{gm} / 1 \\
\text { bigha for 3-6 feet } \\
\text { depth and after 1 } \\
\text { days-1000gm/ } \\
\text { bigha for 3-6 feet } \\
\text { depth }\end{array}$ & ACI & 170 \\
\hline Aquakleen & $\begin{array}{l}\text { Tetradesailtrymethyl, } \\
\text { ammonium, BKC, Amino } \\
\text { nitrogenet }\end{array}$ & $\begin{array}{l}\text { To protect from } \\
\text { various microbes }\end{array}$ & $\begin{array}{l}0.5 \mathrm{~L} / \mathrm{acre} \\
\text { nacre }\end{array}$ & $\begin{array}{l}\text { Square } \\
\text { Pharmaceuti } \\
\text { cals Ltd. }\end{array}$ & $432 / \mathrm{L}$ \\
\hline
\end{tabular}

\subsubsection{Oxygen supplier}

Oxydizing agent, hydrogen peroxide are major active ingredients of oxygen supplier. Oxygen supplier used in the study area is shown in Table 7.

Table 7. List of Oxygen supplier used in pond of study area.

\begin{tabular}{|c|c|c|c|c|c|}
\hline Trade name & $\begin{array}{l}\text { Active } \\
\text { ingredients }\end{array}$ & Purposes & Dose & Source & Price (Taka) \\
\hline Oxygold & $\begin{array}{l}\mathrm{Na}_{2} \mathrm{Co}_{3}, \mathrm{H}_{2} \mathrm{O}_{2^{-}} \\
90 \%\end{array}$ & $\begin{array}{l}\text { Produce/Supply } \\
\text { Oxygen }\end{array}$ & $\begin{array}{l}\text { 750-1000 } \\
\text { gm/acre }\end{array}$ & Fishtech (BD) Ltd. & $670 / \mathrm{kg}$ \\
\hline Oxygrow & $\begin{array}{l}10 \% \text { Hydrogen } \\
\text { peroxide }\end{array}$ & $\begin{array}{l}\text { To supply } \\
\text { sustained Oxygen } \\
\text { for fish }\end{array}$ & $\begin{array}{l}250-300 \\
\text { gm/acre/3-6 feet } \\
\text { depth (Two times } \\
\text { weekly) }\end{array}$ & $\begin{array}{l}\text { Novartis Anmal } \\
\text { Health Ltd. }\end{array}$ & 200 \\
\hline Oxylife & $\begin{array}{l}\text { Oxygen } \\
\text { precursors, } \\
\text { probiotic and } \\
\text { detoxificant }\end{array}$ & $\begin{array}{l}\text { To remove toxic } \\
\text { gases by aerobic } \\
\text { decomposition of } \\
\text { organic wastages }\end{array}$ & $\begin{array}{l}400 \mathrm{gm} / \mathrm{acre}(3-6 \\
\text { feet depth) }\end{array}$ & $\begin{array}{l}\text { Square } \\
\text { Pharmaceuticals } \\
\text { Ltd. }\end{array}$ & 610 \\
\hline Bio-Ox & $\begin{array}{l}\text { Sodium per } \\
\text { carbonate }\end{array}$ & $\begin{array}{l}\text { To supply } \\
\text { Oxygen }\end{array}$ & $10 \mathrm{gm} / \mathrm{dec}$ & ACI Animal Health & $475 / \mathrm{kg}$ \\
\hline Bio Care & $\begin{array}{l}\text { Sodium Lorile } \\
\text { Ether Sulphate }\end{array}$ & $\begin{array}{l}\text { To supply } \\
\text { Oxygen }\end{array}$ & $\begin{array}{l}80-120 \mathrm{ml} / 100 \\
\mathrm{dec}\end{array}$ & ACI Animal Health & $300 / \mathrm{L}$ \\
\hline Oxyren & $\begin{array}{l}\text { Sodium } \\
\text { Carbonate per } \\
\text { Oxyhydrate } \\
\end{array}$ & $\begin{array}{l}\text { To help of the } \\
\text { decomposition of } \\
\text { organic wastages }\end{array}$ & $\begin{array}{l}500-700 \mathrm{gm} / \text { acre } \\
\text { (3 feet depth) }\end{array}$ & Renata Ltd. & $610 / \mathrm{kg}$ \\
\hline
\end{tabular}

\subsubsection{Growth promoter}

There are several chemicals found in the chemical shops which were used as growth promoter as well as to raise production of fish (Table 8). 
Table 8. List of growth promoter used in pond of study area.

\begin{tabular}{|c|c|c|c|c|c|}
\hline Trade name & $\begin{array}{l}\text { Active } \\
\text { ingredients }\end{array}$ & Purposes & Dose & Source & Price (Taka) \\
\hline Aquaboost & $\begin{array}{l}\text { Organic acid, } \\
\text { betaglucan }\end{array}$ & $\begin{array}{l}\text { To increase the } \\
\text { immunity of } \\
\text { fishes, To keep } \\
\text { good health of } \\
\text { fishes }\end{array}$ & $50 \mathrm{gm} / \mathrm{kg}$ & $\begin{array}{l}\text { Novartis Anmal } \\
\text { Health Ltd. }\end{array}$ & $670 / \mathrm{kg}$ \\
\hline Charger Gel & $\begin{array}{l}\text { 1-3D-glucan, } \\
\text { polysaccharide, } \\
\text { Betain }\end{array}$ & $\begin{array}{l}\text { To keep good } \\
\text { health of fishes }\end{array}$ & $2-4 \mathrm{gm} / \mathrm{kg}$ & $\begin{array}{l}\text { Fishtech (BD) } \\
\text { Ltd. }\end{array}$ & $1060 / \mathrm{kg}$ \\
\hline Aqua Gel & $\begin{array}{l}\text { EAA, Fatty acid, } \\
\text { minerals, } \\
\text { antioxydent, } \\
\text { enrich binder gel }\end{array}$ & $\begin{array}{l}\text { To improve the } \\
\text { tast of feed for } \\
\text { better } \\
\text { consumption }\end{array}$ & $\begin{array}{l}\text { In prevention 5- } \\
10 \mathrm{gm} / \mathrm{kg} \text { feed per } \\
\text { day,In treatment } \\
10-15 \mathrm{gm} / \mathrm{kg} \text { feed } \\
\text { per day for } 7-10 \\
\text { days }\end{array}$ & $\begin{array}{l}\text { Square } \\
\text { Pharmaceuticals } \\
\text { Ltd. }\end{array}$ & $560 / \mathrm{kg}$ \\
\hline $\begin{array}{ll}\text { Acimix } & \text { super } \\
\text { Fish } & \end{array}$ & $\begin{array}{l}\text { Vitamin, } \\
\text { Mineral, Amino } \\
\text { acid }\end{array}$ & $\begin{array}{l}\text { To improve } \\
\text { growth of fish }\end{array}$ & $2.5 \mathrm{gm} / \mathrm{kg}$ feed & $\begin{array}{l}\text { ACI Animal } \\
\text { Health }\end{array}$ & $325 / 2.5 \mathrm{~kg}$ \\
\hline Aquamin & Methionin, Lysin & $\begin{array}{l}\text { To improve the } \\
\text { tast of feed }\end{array}$ & $2-4 \mathrm{gm} / \mathrm{kg}$ feed & $\begin{array}{l}\text { ACI Animal } \\
\text { Health }\end{array}$ & $140 / \mathrm{kg}$ \\
\hline Rena fish & $\begin{array}{l}\text { Vit } \\
\text { A,B,C,D3,E,K, } \\
\mathrm{Cu}, \mathrm{Mn}, \mathrm{Fe}, \mathrm{Co} \\
\text { etc }\end{array}$ & $\begin{array}{l}\text { To keep good } \\
\text { health of fishes }\end{array}$ & $1 \mathrm{~kg} / \mathrm{ton}$ feed & Renata Ltd. & $260 / \mathrm{kg}$ \\
\hline Megavit-Aqua & $\begin{array}{l}\text { Vitamin A, Ca, } \\
\text { P, Na etc }\end{array}$ & & $\begin{array}{l}100 \mathrm{gm} / 100 \mathrm{~kg} \\
\text { feed }\end{array}$ & $\begin{array}{l}\text { Novartis Anmal } \\
\text { Health Ltd. }\end{array}$ & $360 / \mathrm{kg}$ \\
\hline
\end{tabular}

\subsubsection{Chemicals for disease treatment}

Most farmers use Virex, Ablaze, Salt, Sumithion, Methylene Blue, Malchite Green in the treatment of fish diseases. Mainly these were used to protect fishes from bacterial, viral and other fungal diseases (Table 9).

Table 9. List of disease treatment chemicals used in pond of study area.

\begin{tabular}{|c|c|c|c|c|c|}
\hline Trade name & Active ingredients & Purposes & Dose & Source & Price (Taka) \\
\hline Ablaze & $\begin{array}{l}\text { Doxycycline- } \\
\text { 10000mg, Colistine } \\
\text { Sulphate- } 1000 \mathrm{mg}\end{array}$ & $\begin{array}{l}\text { In prevention } \\
\text { single time in } \\
\text { monthly }\end{array}$ & $\begin{array}{l}\text { Mainly used in } \\
\text { the treatment of } \\
\text { lesion }\end{array}$ & $\begin{array}{l}\text { Eon } \\
\text { Pharmaceutical } \\
\text { Ltd. }\end{array}$ & 390 \\
\hline Salt & $\mathrm{Nacl}$ & $\begin{array}{l}\text { For eradication of } \\
\text { bacterial and } \\
\text { external parasites }\end{array}$ & $\begin{array}{l}2.5 \% \text { for } 20-30 \\
\text { minute repeat in } \\
48 \text { hours }\end{array}$ & Chemical seller & $12 / \mathrm{kg}$ \\
\hline Methylene Blue & $\mathrm{C}_{10} \mathrm{H}_{18} \mathrm{CIN}_{3} \mathrm{SH}_{2} \mathrm{O}$ & $\begin{array}{l}\text { To eradicate } \\
\text { bacteria and } \\
\text { fungus }\end{array}$ & $3 \mathrm{ppm}$ & Chemical seller & $350 / 25 \mathrm{gm}$ \\
\hline Malchite Green & $\mathrm{C}_{2} \mathrm{H}_{2} \mathrm{O}_{4}$ & $\begin{array}{l}\text { To eradicate } \\
\text { fungus and cyst of } \\
\text { parasites }\end{array}$ & $0.15 \mathrm{ppm}$ & Chemical seller & $500 / 25 \mathrm{gm}$ \\
\hline Sumithion & Active sumithion & $\begin{array}{l}\text { To eradicate } \\
\text { copepods and } \\
\text { crustacean } \\
\text { parasites of fishes }\end{array}$ & $0.25-0.50 \mathrm{ppm}$ & Chemical seller & $60 / 100 \mathrm{ml}$ \\
\hline
\end{tabular}

\subsubsection{Gas reducer}

The present study identified a range of chemicals were used to reduce gases and to maintain the proper quality (Table 10). 
Table 10. List of Gas reducer chemical used in pond of study area.

\begin{tabular}{|c|c|c|c|c|c|}
\hline Trade name & $\begin{array}{l}\text { Active } \\
\text { ingredients }\end{array}$ & Purposes & Dose & Source & Price (Taka) \\
\hline Gastrap & $\begin{array}{l}\text { Biologically } \\
\text { derived } \\
\text { scavengers, } \\
\text { symbiotic in a } \\
\text { fortified powder } \\
\text { base }\end{array}$ & $\begin{array}{l}\text { To provide } \\
\text { oxygen, To } \\
\text { increase growth } \\
\text { and production }\end{array}$ & 200gm/acre & $\begin{array}{l}\text { Square } \\
\text { Pharmaceuticals } \\
\text { Ltd. }\end{array}$ & $300 / \mathrm{kg}$ \\
\hline Ammonil & $\begin{array}{l}\text { Yucca plant } \\
\text { extract Bacilus } \\
\text { subtilis, Micro } \\
\text { encapsulated } \\
\text { enzyme }\end{array}$ & $\begin{array}{l}\text { To remove } \\
\text { ammonia and } \\
\text { other harmful } \\
\text { gases }\end{array}$ & 200gm/acre & $\begin{array}{l}\text { Novartis Anmal } \\
\text { Health Ltd. }\end{array}$ & $580 / \mathrm{kg}$ \\
\hline Biopond & Zeolite, Probiotic & $\begin{array}{l}\text { To reduce toxic } \\
\text { gases in pond }\end{array}$ & $\begin{array}{l}2-3 \mathrm{~kg} / \text { acre in } \\
\text { pond preparation, } \\
1-1.5 \mathrm{~kg} / \text { acre } \\
\text { cultural pond }\end{array}$ & $\mathrm{SK}+\mathrm{F}$ (BD) Ltd. & $425 / \mathrm{kg}$ \\
\hline
\end{tabular}

\subsubsection{Vitamin}

Vitamin and minerals are important ingredients for growth of fish. Four vitamins with different trade names were found to use by the farmers (Table 11).

Table 11. List of vitamins used in pond of study area.

\begin{tabular}{|l|l|l|}
\hline Trade Name & Dose & Source \\
\hline Grow fast & $1 \mathrm{ml} / 3-4 \mathrm{~L}$ & Rals \\
\hline Nutrigrow & $\begin{array}{l}0.5-1 \mathrm{ml} / \mathrm{ton} \text { of water for hatchery, 0.5-10ml/kg of nursery } \\
\text { pond }\end{array}$ & $\begin{array}{l}\text { Sciencetech Agro Industries } \\
\text { Ltd. }\end{array}$ \\
\hline Ossi C & $4-5 \mathrm{gm} / \mathrm{kg}$ feed for 5-7 days & Fish tech \\
\hline Energy Sea & $\begin{array}{l}3-5 \mathrm{gm} / \mathrm{L} \text { for transport of large fish, } 1000 \mathrm{gm} / 33 \mathrm{dec} \text { before } \\
\text { stocking of seed }\end{array}$ & $\begin{array}{l}\text { Sciencetech Agro Industries } \\
\text { Ltd. }\end{array}$ \\
\hline
\end{tabular}

\subsubsection{Enzyme}

Only one type of enzyme was used by the farmers in fish feed named Biozyme. They use it at a dose of 25-50 $\mathrm{gm} / 100 \mathrm{~kg}$ feed. Fish Tech provides the enzyme to the farmers.

\subsubsection{Predator killer}

Farmers used rotenone powder to remove predator fish. Rotenone with a brand name Acurte Gold is provided by a pharmaceutical company Samco was found in the study area. The recommended dose of Acurte Gold was $15 \mathrm{gm} /$ decimal $/ 1 \mathrm{ft}$ depth.

\subsection{Impact of various agrochemicals on fish health and diseases of fish in the study area}

EUS was detected in Tilapia, Rui, Catla and Mrigal with $70 \%$ prevalence. Indian major carps found to be susceptible with Lerneasis having $80 \%$ prevalence. Pangus were also affected by tail rot and fin rot diseases with having $60 \%$ prevalence (Table 12 ). 
Table 12. Impact of various aqua drugs and agrochemicals on fish health and diseases recovery of fish in the study area.

\begin{tabular}{|l|l|l|l|l|l|}
\hline Species & $\begin{array}{l}\text { Diseases } \\
\text { prevalence }\end{array}$ & Clinical sign & Agrochemical used & $\begin{array}{l}\text { Affected } \\
\text { month }\end{array}$ & Recovery \\
\hline Rui, Sarputi & EUS (60\%) & Red spot body surface & $\begin{array}{l}\text { Renamycine 50mg/kg } \\
\text { body weight }\end{array}$ & August & $80 \%$ \\
\hline $\begin{array}{l}\text { Rui, Catla, } \\
\text { Mrigal }\end{array}$ & $\begin{array}{l}\text { Ammonia } \\
\text { poisoning (60\%) }\end{array}$ & $\begin{array}{l}\text { Red or bloody gill, } \\
\text { gasping at the air }\end{array}$ & $\begin{array}{l}\text { Regular water testing } \\
\text { and maintaince }\end{array}$ & January & $90 \%$ \\
\hline $\begin{array}{l}\text { Tilapia,Rui, } \\
\text { Catla, Mrigal }\end{array}$ & EUS (60\%) & Red spot body surface & Antibiotics prevent it & August & $85 \%$ \\
\hline Rui, Catla & $\begin{array}{l}\text { Oxygen } \\
\text { deficiency (70\%) }\end{array}$ & $\begin{array}{l}\text { Gasping at the surface } \\
\text { with their mouth }\end{array}$ & $\begin{array}{l}\text { Oxyflow, Oxylife, } \\
\text { Bio-ox }\end{array}$ & December & $90 \%$ \\
\hline Shing & Black spot (30\%) & $\begin{array}{l}\text { Small black spot on } \\
\text { surface }\end{array}$ & $\begin{array}{l}\text { Use commercially } \\
\text { available drugs }\end{array}$ & January & $70 \%$ \\
\hline $\begin{array}{l}\text { Magur, } \\
\text { Shing,Pangus }\end{array}$ & $\begin{array}{l}\text { Tail rot and Fin } \\
\text { rot (40\%) }\end{array}$ & $\begin{array}{l}\text { Destruction of fins or } \\
\text { tail, exposed fin rays }\end{array}$ & $\begin{array}{l}\text { Tetracycline, } \\
\text { Methylene blue }\end{array}$ & February & $85 \%$ \\
\hline $\begin{array}{l}\text { Indian } \\
\text { carps }\end{array}$ & Lerneasis (20\%) & $\begin{array}{l}\text { Fishes are restless and } \\
\text { rub against the side } \\
\text { and the bottom of the } \\
\text { pond }\end{array}$ & $\begin{array}{l}\text { KMnO } \text { pond } \\
\text { treatment at 4 ppm }\end{array}$ & January & $80 \%$ \\
\hline
\end{tabular}

\section{Discussion}

In the present study, it was noticed that among 50 fishermen of my study areas about $75 \%$ farmers having single ownership and $25 \%$ farmers having multiple ownership. About $82 \%$ fishermen had own land and the other farmer about $18 \%$ had culture others land. In my study areas the minimum and maximum value of $0.6 \mathrm{ha}$ and 12.04 ha. The result found in the present study differs from the result reported by Islam et al. (2014) and Asif et al. (2015) who stated that land condition of fisherman. In this study, it is observed that $80 \%$ farmers follow polyculture system and $20 \%$ farmers followed monoculture system. The cultured fish species are Rui, Catla, Pangus, Tilapia, Koi, Sarputi and other carps. Most of the farm in our country followed polyculture and some farm followed monoculture system (Rahman, 2012; Haq et al., 2017; Shajib et al., 2017; Zafar et al., 2017). The cultured fishes were Rui, Catla, Pangus, Tilapia, Koi, Sarputi, common carp, Mrigal and Magur in Jamalpur and Sherpur district. Faruk et al. (2008) observed that the farmer interviewed were male with an average age of 37 years and the average family size 6; another few study in different place of Bangladesh showed the similar results (Islam et al., 2014; Asif et al., 2015; Hossain et al., 2015; Islam et al., 2015; Sharif et al., 2015; Sultana et al., 2015; Ali et al., 2016; Hossain et al., 2016; Vaumik et al., 2017; Razeim et al., 2017; Zaman et al., 2017; Islam et al., 2017; Asif and Habib, 2017 Adhikary et al., 2018a; Mondal et al., 2018a; Mondal et al., 2018b; Adhikary et al., 2018b). But in this study, the interviewed age 34 years old and their average. At present 24 animal health companies were seen to market at field level. In the study area different types of diseases like EUS, tail rot, fin rot, red spot, white spot and dropsy in different fish species mainly in Shing, Koi, Tilapia and Pangus were observed. A number of authors reported the similar condition in aquaculture of Bangladesh (DoF, 2002; Faruk et al., 2004; Asif et al., 2014; Shabuj et al., 2016; Yeasmin et al., 2016; Rahman et al., 2017; Neowajh et al., 2017; Biswas et al., 2018; Rahman et al., 2018). Most of the farmers of the selected areas used drugs and chemicals to control these types of diseases. It was observed that farmers of the study areas go good results in diseases treatment by applying single or combination of commercial aqua drugs and chemicals. In some cases after use of drugs they got about $95 \%$ recoveries within a short period of time. These result influence farmers to use more commercial aqua drugs and chemicals for diseases control and treatment. Normally in rural aquaculture farmers used traditional aqua drugs such as are lime, salt, urea, triple super phosphate (TSP), potassium permanganate, vitamins, antibiotics, (mainly oxytetracycline and chlorotetracycline), rotenone, phostoxin, sumithion, melathion and some hormones. It is also found that most of the farmers used chemicals and antibiotics (Chowdhury et al., 2015; Biswas et al., 2018; Adhikary et al., 2018). In the present study identified range of aqua chemicals and antibiotics marketed by different companies for using in various activities of aquaculture. Fish health management and diseases treatment were the major areas where farmers were seen to use a lot of such compounds. Other uses included growth promotion, improve water quality and as probiotics. Commonly found traditional chemicals in health management including are potassium permanganate, sumithion, melathion, formalin, bleaching powder, malachite green and methylene blue (Chowdhury et al., 2015; Rahman et al., 2017; Biswas et al., 2018; Rahman et al., 2018). Some previous 
studies also revealed the similar reports about the use of chemicals used in aquaculture of Bangladesh (Philips, 1996; Brown and Brooks, 2002; DoF; 2002; Faruk et al., 2004; Chowdhury et al., 2015; Biswas et al., 2018; Adhikary et al., 2018). The water quality management plays an important role in growth, development, reproduction, survival as well as feed consumption. For water quality management agriculture lime, Geotox, JV Zeolite, Mega Zeo Bio, Aquakleen and Biomin pond life have been used in the study area. Farmers use different types of aqua-medicine during pond preparation and water quality improvement. Anwar (2014) observed that for water quality management Geotox, JV Zeolite, Life, Aquakleen, Biomin, Aquabost are used. Chemicals like Geotox, JV Zeolite, Mega Zeo, Lime, BioAqua and Ames Zeolite are repeated by Monsur (2012) for pond preparation and water quality management. Dissolved oxygen is one of the most important factors of pond water for aquaculture practices. Farmers of the investigated areas use several chemicals to increase oxygen concentration in pond like Bio-ox, Oxygold, Oxylife, Oxymas, Oxyren, Oxyflow and Oxysos. Monsur (2012) observed that farmers used Oxyflow, Oxymas, Bio Care, Bio Ox, Oxy-gold and Oxy-A to improve dissolved oxygen level in Jamalpur and Sherpur regions. Faruk et al. (2008) found that the farmers of the Mymensingh region used Bio-ox, Gesonex, Oxymarine, Oxy plus, Oxymas, Oxymore and Bio care to increase dissolve oxygen. Rahman (2011) observed that aqua drugs like Oxyflow, Oxymas, Oxy plus, Bio Care, Pure Oxy, OxyGold were used to increase dissolved oxygen, Oxidizing agent, Hydrogen Peroxide was the major active ingredients of such chemicals. Ali (2008) reported that Oxyflow and Oxymas were used to remove poisonous gases. Several chemicals with almost similar names were seen readily available in the chemical shops to use for increasing dissolved Oxygen (DO) in ponds. The propensity of using harmful gas controller is higher than any other medicines used by farmers in Maheshpur upazila. In the case $36 \%$ farmers showed their tendency to use, because most of the farmers had problems with harmful gases in their ponds (Uddin et al., 2014). Disinfectants are widely used in many spheres of aquaculture. As disinfectants, farmers use Timsen, Polgard plus, Formalin, bleaching Powder, EDTA and Eraprim vet in the study areas. Among them Timsen (disinfectant) is mostly available chemical in the chemical shops. Anwar (2014) mentioned that Hepartect-Aqua, Timsen, virex, Polgard Plus as disinfectant, Lime, Salt, Formalin, Potash and Malchinate Green were used against fish diseases in Jamalpur Sadar Upazila. Rahman (2011) stated that about 10 disinfectants from nine different sources were found to use by the farms in Mukta gasa, Fulbaria and Fulpur of Mymenshing district such as Polgard plus, Bactisal, virex, Biogard, Lenocide, Timsen, Emsen, Aquacleaner Plus, Formalin, Bleaching Powder. Shamsuddin (2012) observed that as disinfectants farmers used mostly Polgard plus, Aquakleen, Bleaching Powder, Bactisal, Virex and Timsen in the Fulbaria, Gouripur and Fulpur Upazilas of Mymenshing district. Seven antibiotics with different trade names are used by the fish farmers in the present study areas as Novamix 101, Erocot, Captor, Oxysentin 20\%, Renamycine, Aquamycine and Oxysentin 20\% are antibiotics with different trade names were seen in the market as well as used by the fish farmers in the study area. All of these antibiotics are effective against bacterial disease. Faruk et al. (2008); Chowdhury et al. (2015); Biswas et al. (2018); Adhikary et al. (2018) observed that several branded antibiotics were found in the market; among them Oxysentin, Renamycine, Renamox and Orgamycine were being widely used. Sarkar et al. (2014) reported that Renamycine, Bactitab, Chlorstechlin, Cotrim- Vet, Orgacycline were being used antibiotics in coastal region of Bangladesh. Monsur (2014) also reported that Antibiotics should be used for bacterial diseases. From the research findings of Shamsuddin (2012), it was reported that most of the farmers used Renamycine at the rate of $100 \mathrm{mg} / \mathrm{kg}$ feed for 7 days for its high activity against bacterial diseases. The present study revealed that commercial aqua drugs have some positive impact on fish health management and diseases treatments at farmer's level. The present study identified arrange aqua chemicals and antibiotics market by different companies for using in various activities of aquaculture fish health management and disease treatment where the measure areas farmers sin to use a lot of such compounds. The present study also noticed a number of new product with various trade names in the market which is included zeolite, Geotox, mega geo, Aqua boost, Oxyflow, Biotuff quick oxygen, Orgavit, Aqua, Aqua Gold, Timsen, Efinol etc us video and various antibiotics and probiotics. The local animal feed and chemicals shops are the main source options compound from where farmers usually purchased these. The number of authors also reported similar condition in aquaculture of Bangladesh (Amin, 2000; Faruk et al. 2004). Growfast, Ossi-C, Nutrigrow are found in the study areas. Shamsuddin (2012) observed that farmers used Grow fast, Ossi-C, Silver mil and Revit C were used as a growth promoter in Fulbaria, Gouripur and Faridpur upazila of Mymenshing. Faruk et al. (2008) also reported that farmers used growth promoter such as Megavit Aqua, Aqua Boost, Aqua Savor and Vitamin premix, Grow fast, Vitamix and Grow max in Jhenaidah district. Farmers used as growth promoters Megavit Aqua, Aqua Boost, Aqua Savor, Vitamin premix, Grow fast, Nutricell Aqua, Rapid Aqua, Cevit Vet, Acimix super fish etc. In the study area only few people farmers used growth promoters increase diseases resistance power of fish because of high price. Various types of diseases are observed in Maheshpur Upazila. August, December, January and 
February are susceptible months of diseases outbreak in the study area. Monsur (2012) found that the affected month was August, November, January and February in Jamalpur and Sherpur district. However in February and March fish were also affected by diseases. In the study area some kinds of diseases were founded such as Epizotic Ulcerative Syndrome (EUS), skin diseases, gill damage, tail and fin rot. For highest stocking density tilapia and pangus were mostly affected by diseases in winter. In the study area different types of diseases like EUS, tail rot, fin rot, red spot, white spot and dropsy in different fish species mainly in Shing, Koi, Rui, Catla, Tilapia and Pangus were observed. A number of authors reported the similar condition in aquaculture of Bangladesh (Faruk et al. 2004). Most of the farmers of the selected areas used drugs and chemicals to control these types of diseases. It meant that's diseases problem was one of the major concern in aquaculture of the studied areas. Alam and Rashid (2014) mentioned that Tilapia (Oreochromis nilotika), Sharputi (Puntius sarana) Rui (Labeo rohita), Catla (Catla catla), Mrigal (Cirrhinus cirrhosus), Bagda (Penaeus monodon), Golda (Macrobachium rosenbergi), and Silver carp (Hypophthalmicthys molitrix) were affected by various types of diseases such bacterial infection, EUS (Epizootic Ulcerative Syndrome), ichthyophthiriasis, argulosis, swollen abdomen and white spot diseases in Shatkhira district. Anwar (2014) reported that EUS (Epizotic Ulcerative Syndrome), red spot and different parasitic diseases occurred in Pangus (Pangasianolan hypohthalmus), Tilapia, Sharputi, Rui, Mrigal, Thai koi (Anabas testudineus), Shing (Heteropneustes fossilis) and silver carp in Jamalpur Sadar Upazila. Monsur (2012) observed that in Jamalpur and Sherpur district farmers used mainly Renamycine, Polgrad plus, Ossi-C, Timsen, Bactisal and Aquamycine for the fish disease treatment. Prasad et al. (1996) found that chloramphenicol and oxytetracycline would be effective drug in curing the EUS lesion tetracycline and streptomycine were found to be less effective in curing the ulcers. The present study identifies that, in case of rui, sarputi Renamycine $50 \mathrm{mg} / \mathrm{kg}$ body weight are effectively used in curing the EUS. Farmers use Renamycin with a result of $80-85 \%$ recovery to treat EUS in rui, sarputi, tilapia in the study area. But Rahman (2011) mentioned that EUS affected tilapia was treated with Renamycin, Polgard plus and Ossi C with 80-95\% recovery form disease in Fulpur and Muktagacha. About 52\% farmers used potassium permanganate, while $40 \%$ used lime, $11 \%$ used salt as a disease treatment in Bangladesh. According to the reports of Brown and Brooks (2002), a few Farmers used other treatments such as disinfectants, banana leaves, fertilizers, alum exchange. The present study identifies that tail rot and fin rot affected pungus are treated with tetracycline, methylene blue having 85\% recovery. Rahman (2011) observed that Edwardsiellosis affected pangus were treated with renamycine, Timsen, Polgard plus, Ossi C in Fulpur and with Geolite and Timsen in Fulbaria having $80 \%$ recovery in both upazilas.

\section{Conclusions}

In conclusion, the present study demonstrated current status of chemicals and antibiotics using in aquatic animal health management and pointed out some problems of the use of chemicals by the farmers who is include lack of knowledge of the chemicals, doses and method of application of these chemicals. There are few alternatives to minimize the adverse effect of aquaculture chemicals simply use less of them. Other alternatives could be used as bioremediation and use of probiotics immune stimulants vaccination and alternative therapeutic. However policy maker researches and scientist should work together in addressing the issues of chemical used in aquaculture with a view to reduce the negative impacts.

\section{Conflict of interest}

None to declare.

\section{Reference}

Adhikary MR, MA Rahman, AA Asif and RK Adhikary, 2018b. Socio-economic status of fish retailers in Jashore sadar, Bangladesh. Asian Australas. J. Food Saf. Secur., 2: 100-108.

Adhikary RK, M Rahman and AA Asif, 2018. Present status of aqua-medicines used in aquaculture at Jessore sadar upazila, Bangladesh. Asian J. Med. Biol. Res., 4: 288-297.

Adhikary RK, S Kar, A Faruk, A Hossain, MNM Bhuiyan and AA Asif, 2018a. Contribution of aquaculture on livelihood development of fish farmer at Noakhali, Bangladesh. Asian Australas. J. Biosci. Biotechnol., 3:106-121.

Alam MA and MM Rashid, 2014. Use of aqua- medicines and chemicals in aquaculture in Shatkhira District, Bangladesh. J. Pharm. Biol. Sci., 9: 5-9.

Ali MM, 2008. Study on the chemicals and antibiotics used in aquatic animal health management. MS Thesis, Department of Aquaculture, Bangladesh Agriculture University, Mymensingh, Bangladesh. 
Ali MM, AA Asif, MAI Shabuj, S Vaumik, MA Zafar and BMN Sharif, 2016. Status of polyculture Pangasius hypophthalmus with carps in Jhikargacha Upazila of Jessore District, Bangladesh. Int. J. Fish. Aqua. Stud., 4: 423-430.

Anwar MA, 2014. Aqua drugs and chemicals used in aquaculture in Jamalpur Sadar Upazila. Department of Aquaculture, Bangladesh Agricultural University, Mymensingh.

Apu NA, 2014. Farmed fish value sends development in Bangladesh: situation analysis and trends. World Fish/ILRI project report. Nairobi Kenya, International Livestock Research Institute (ILRI).

Asif AA and MAB Habib, 2017. Socio-economic condition of fish farmers of Jhikargachha upazila in Jessore district, Bangladesh. Asian J. Med. Biol. Res., 3: 462-475.

Asif AA, MA Samad, BMS Rahman, MA Rahman, MH Rahman, SM Yeasmin, and A Nima, 2014. Study on management of fish fry and fingerling marketing of Jessore in Bangladesh. Int. J. Bus. Soc. Sci. Res., 2: 127135.

Asif AA, MA Samad, MH Rahman, MA Farid, SM Yeasmin and BMS Rahman, 2015. Socio-economic condition of fish fry and fingerling traders in greater Jessore region, Bangladesh. Int. J. Fish. Aqua. Stud., 2: 290-293.

BFRI, 1999. Fish disease prevention and control, Bangladesh fisheries research institute, circular leaflet no. 6.2nd edition. pp.7.

Biswas C, MMM Hossain, AA Asif, B Sarker, MM Billah and MA Ali, 2018. Culture strategies, diseases and their mitigations in mono-sex Nile tilapia farming in Jessore sadar region, Bangladesh. Asian Australas. J. Biosci. Biotechnol., 3: 190-200.

Brown D and A Brooks, 2002. A survey of disease impact and awareness in pond aquaculture in Bangladesh,the fisheries and training extension project phase: Arther, J,R,M.J. Philips.R.P.Subasinghe, M.B.Reantaso and I.H.Mac Rae(eds). Primary aquatic animal health care in rural, small scale and aquaculture development, FAO Fisheries Technology Paper 406: 85-93.

Chowdhury AA, MS Uddin, S Vaumik and AA Asif, 2015. Aqua drugs and chemicals used in aquaculture of Zakigonj upazilla, Sylhet. Asian J. Med. Biol. Res., 1: 336-349.

Faruk MAR, MJ Alan, MB Kobir, 2004a. Status of fish disease and health management practices in rural freshwater aquaculture of Bangladesh. Pakistan J. Biol. Sci., 7: 2092-2098.

Faruk MAR, MJ Alan, MB Kobir, 2004b. Economic loss from fish disease on rural freshwater aquaculture Bangladesh. Pakistan J. Biol. Sci., 7: 2086-2091.

Faruk MAR, MMR Sarkar, ZP Patwary, 2008. Evaluation of the status of use of chemicals and antibiotics in freshwater aquaculture activities with special emphasis to fish health management. J. Bangladesh Agric. Univ., 6: 381-390.

Faruk MAR, N Sultana and MB Kabir, 2005. Use of chemicals in aquaculture activities in mymensingh area, Bangladesh. Bangladesh J. Fish., 29: 1-10.

Haq ME, M Rahman, A Hossain, AA Asif, H Rahman, P Chwakravorty, A Satter and MS Islam, 2017. Comparative growth performance between monosex and natural XY male tilapia in Noakhali region, Bangladesh. Asian J. Med. Biol. Res., 3: 391-397.

Hossain MA, AA Asif, MA Zafar, MT Hossain, MS Alam and MA Islam, 2015. Marketing of fish and fishery products in Dinajpur and livelihoods of the fish retailers. Int. J. Fish. Aqua. Stud., 3: 86-92.

Hossain MZ, A Pal, MA Hasan, MS Parvej, N Nahar and AA Asif, 2016. Nutritional status and sociodemographic characteristics of the people of south-west coastal region in Bangladesh. Asian Australas. J. Biosci. Biotechnol., 1: 323-332.

Islam FMK, AA Asif, M Ahmed, MS Islam, B Sarker, MA Zafar and M Rahman, 2017. Performances of resource poor households in aquaculture practices in sadar upazila, Meherpur, Bangladesh. Int. J. Fish. Aqua. Stud., 5: 281-288.

Islam MA, AA Asif, MA Samad, BMS Rahman, MH Rahman, A Nima and SM Yeasmin, 2014. Socioeconomic conditions of the fish farmers in Jessore, Bangladesh. Int. J. Bus. Soc. Sci. Res., 2: 153-160.

Islam MM, AA Asif, S Vaumik, MA Zafar, BMN Sharif, MH Rahman and S Shahriyar, 2015. Socio economic status of fry collectors at Sundarban region. Int. J. Fish. Aqua. Stud., 3: 89-94.

Karim M and J Stellwagen, 1998. Final report on 4th fisheries projects: shrimp aquaculture (Preparatory phase for National Development program). Department of Fisheries, Ministry of Fisheries and Livestock, Bangladesh.

Mahmud AN, MR Hasan, MB Hossain and MH Minar, 2012. Proximate composition of fish feed ingredients available in in lakshimpur region. American Euro Asian J. Agric. Env. Sci., 12: 556- 560. 
Meisinger JJ and JA Delago, 2002. Principles of managing nitrogen leaching. J. Soil Water Conser., 57: 485489.

Mondal MAI, MA Kader, AH Choudhury, MG Mustafa, MRU Nabi, MM Billah, AA Asif and AAM Siddiqui, 2018a. Socio-economic uplifting analysis of ESBN fishery of the coastal villages, Kumira and Kattoli, Chittagong, Bangladesh. Int. J. Res. Grant., 6: 248-263.

Mondal MAI, MA Kader, MRU Nabi, AAM Siddiqui, MM Billah and AA Asif, 2018b. Bio-economic analysis of ESBN fishery of Kumira, the coastal area of Chittagong, Bangladesh. Asian J. Med. Biol. Res., 4: 315322.

Monsur A, 2012. Use of Aqua drugs and chemicals of aquaculture in Jamalpur and Sherpur Regions. MS thesis, Department of Aquaculture, Bangladesh Agricultural University, Mymensingh, Bangladesh.

Neowajh MS, MM Rashid, AA Asif, MA Zafar and A Hossain, 2017. Effects of chemotherapeutics against experimentally injured stinging catfish Heteropneustes fossilis. Asian J. Med. Biol. Res., 3: 476-487.

Philips M, 1996. The use of chemicals in carp and shrimp aquaculture in Bangladesh, Cambodia, Nepal, Pakistan, Sri Lanka and Vietnam. In: use of chemicals in aquaculture in Asia. Arthur JR, southeast Asian fisheries development centre aquaculture department Tigbauan, IIoilo, Philippines. pp. 75-84.

Prasad Y, SA Mastan and CH Samuel, 1996. Effect of different antibiotic on EUS affected fish Channa striatus. Asian Fish. Soc., pp.191.

Primavera JH, LCR Pitogo and DMR Pena, 1993. A survey of chemical and biological products used in intensive prawn farms in the Philippines. Mar. Poll. Bull., 26: 35-40.

Rahman H, 2012. Effects of aqua drugs on the health condition of farm fishes of Jamalpur areas. MS thesis, Department of Aquaculture, Bangladesh Agricultural University, Mymensingh, Bangladesh.

Rahman MA, MH Rahman, Yeasmin SM, AA Asif and D Mridha, 2017. Identification of causative agent for fungal infection and effect of disinfectants on hatching and survival rate of bata (Labeo bata) larvae. Adv. Plant. Agric. Res., 7: 00264.

Rahman MH, SM Yeasmin, MA Rahman, MA Farid and AA Asif, 2019. Effect of formalin on fertilization, hatching rate of eggs of Thai Pangas (Pangasius hypophthalmus) and survival and growth performance of fry. Int. J. Biosci., 14: 158-165.

Rahman MM, 2011. Status and impact of commercial aqua drugs and chemicals on his health at farmer's level. MS thesis, Department of Aquaculture, Bangladesh Agricultural University, Mymensingh.

Razeim MA, MG Farouque, MA Sarker, AA Asif and M Ahmed, 2017. Attitude of farmers towards Pangas farming for their livelihood improvement. Asian Australas. J. Biosci. Biotechnol., 2: 106-119.

Sarkar MR, MR Mokhlesur, MM Ali and MC Reaz, 2014. Drugs and chemicals used in aquaculture activities for fish health management in the coastal region of Bangladesh. Int. J. Life Sci. Biotech. Pha. Res., 3: 40-45.

Shabuj MAI, T Bairagi, AA Asif, O Faruq, MR Bari and MS Neowajh, 2016. Shrimp disease investigation and culture strategies in Bagerhat district, Bangladesh. Asian J. Med. Biol. Res., 1: 545-552.

Shajib MSH, B Sarker, AA Asif, MM Rahman, MA Zafar and A Hossain, 2017. Effects of stocking density on the growth rate of gold fish fry reared in hapa. Asian J. Med. Biol. Res., 3: 504-515.

Shamsuddin M, 2012. Impact of aqua drugs and chemicals on health and production of fish. MS thesis, Department of Aquaculture, Bangladesh Agricultural University, Mymensingh, Bangladesh.

Sharif BMN, AA Asif, S Vaumik, MA Zafar, MM Islam and MA Samad, 2015. Socio-economic condition of fish farmer and trader at the village of Pitamborpur in Chaugachha upazilla in Jessore, Bangladesh. Int. J. Fish. Aqua. Stud., 3: 212-217.

Subasinghe RP, U Barg and A Tacon, 1996. Chemicals in Asian aquaculture need,Usage, Issues and challenges.In: use of chemicals in aquaculture in Asia.Arthur JR, Pitogo LCR, Subasinghe RP. South east Asian Fisheries Development Centre, Aquaculture Department Tigbauan,IIoilo, Philippines. pp.1-6.

Sultana N, AA Asif, MMI Dihider, SM Ahsan and FS Maraj, 2015. Usefulness of farm women training programmes in livelihood security. Int. J. Bus. Soc. Sci. Res., 4: 13-24.

Uddin MM, BB Sarkar and US Tonny, 2014, Propensity e of using harmful gas controller and oxygen supplier on the basis of fish farmers age, educational status and land ownership of six Upazilas in Noakhali district Bangladesh. Global J. Sci. Fro. Res. 14: 2249- 4626.

Vaumik S, SK Sarker, MS Uddin, MT Alam, A Satter and AA Asif, 2017. Constraints and prospects of fish farming in Lalmonirhat District. Int. J. Bus. Soc. Sci. Res., 5: 201-210.

Yeasmin SM, MA Rahman, MMM Hossain, MH Rahman and AA Asif, 2016. Identification of causative agent for fungal infection and effect of disinfectants on hatching and survival rate of common carp (C. carpio) larvae. Asian J. Med. Biol. Res., 1: 578-588. 
Zafar MA, MZ Hasan, MM Ali and AA Asif, 2017. Growth and production performance of Vietnamese koi (Anabas testudineus) with Magur (Clarias batrachus) at different stocking densities. Asian-Australasian J. Biosci. Biotech., 2: 226-237.

Zaman MFU, MA Samad, MA Islam, MHU Jaman, S Khondoker and AA Asif, 2017. Assessment of sustainability of Pangasius (Pangasius hypophthalmus) farming at Jhikargachha upazila in Jessore district, Bangladesh. Int. J. Fau. Biol. Stud., 4: 109-119. 